\title{
Hábitos alimentarios del Puma concolor (Carnivora: Felidae) en el Parque Nacional Natural Puracé, Colombia
}

\author{
Andrés Hernández-Guzmán ${ }^{1}$, Esteban Payán ${ }^{2}$ \& Octavio Monroy-Vilchis ${ }^{3}$ \\ 1. Grupo de Estudios en Geología Ecología y Conservación (GECO), Universidad del Cauca, Apartado postal 082 \\ Popayán-Cauca, Colombia; andreshguzman@hotmail.com \\ 2. Panthera, Carrera 7 N. ${ }^{\circ}$ 156-78, oficina 1004, Bogotá; epayan@panthera.org \\ 3. Estación Biológica Sierra Nanchititla, Facultad de Ciencias, Universidad Autónoma del Estado de México. Instituto \\ Literario 100, Colonia Centro, 50000, Toluca, México; omv@uaemex.mx
}

\section{Recibido 23-IV-2010. Corregido 04-I-2011. Aceptado 26-I-2011.}

\begin{abstract}
Food habits of Puma concolor (Carnivora: Felidae) in the Parque Nacional Natural Puracé, Colombia. Neotropical puma (Puma concolor) diet is scarcely known, in particular that of mountain dwelling individuals from Northern South America. This is the first study on pumas from the paramo and the first puma diet analysis for Colombia. The puma diet was studied from 2007 to 2009 in the Puracé National Park in the South Colombian Andes. Paramos are unique neotropical high altitude ecosystems which store and regulate water, and are currently threatened by agricultural expansion and climate change. Seven latrines were monitored for three years and scat collected, washed and dried. Items in scat such as hair, bones, claws and others were separated. Hairs were inspected by microscopy and compared to voucher hair museum specimens. Bone fragments, claws and teeth were also compared to museum collections and identified wherever possible. Additionally, six cameras were set along game trails to document puma and potential prey presence in the area. Food items from five species were identified in 60 puma scats; Northern Pudu (Pudu mephistophiles) was the most important prey in their diet. A total of 354 camera trap-nights photographed a male and female puma, Northern pudu and Spectacled bear (Tremarctos ornatus). The main conclusion suggests a strong dependence of puma on the threatened and mysterious Northern Pudu in paramo habitats. This behavior might reflect restricted prey availability in the high Andes mountains of Colombia, and highlights the plasticity in the puma diet. Conservation actions in the paramo should thus, focus on focal wild species, and in particularly those that show a relationship, such as the one evidenced here with the dependence of puma on Northern Pudu. These findings contribute to increase the little known ecology of Andean puma populations and the species as a whole in Colombia. Baseline data on puma prey populations in different ecosystems throughout their range, is critical to understand the regional requirements for survival, and design conservation actions, to follow and evaluate the need for particular protected areas along their geographical gradients. Rev. Biol. Trop. 59 (3): 1285-1294. Epub 2011 September 01.
\end{abstract}

Key words: Colombian Andes, camera traps, diet, prey, Pudu, Puma concolor, Puracé National Park.

El Puma concolor (Linneaus 1758), una especie casi amenazada (Caso et al. 2008) es el segundo felino más grande en América y la subespecie para Colombia es Puma concolor concolor (Culver et al. 2000). El puma, es el mamífero con más amplia distribución en el hemisferio occidental (Shaw et al. 2007, Laundré \& Hernández 2010) y ha sido encontrado desde el norte de América hasta el sur de Argentina (Beck et al. 2005, Shaw et al. 2007). La densidad del puma en Norteamérica es de aproximadamente dos individuos adultos por $100 \mathrm{~km}^{2}$ (Nowell \& Jakson 1996) y en algunos sitios del Centro de México entre 2.74$6.86 / 100 \mathrm{~km}^{2}$ (Soria-Díaz et al. 2010); mientras que para Bolivia (6.80 \pm 1.5$)$, Belice (3.42 \pm 1.3 ) 
y Argentina (0.67 \pm 0.2$)$, los estudios muestran que a lo largo de su distribución, la densidad puede variar considerablemente entre sitios (Kelly et al. 2008, Laundré \& Hernández 2010).

La flexibilidad del comportamiento del puma es un importante mecanismo de supervivencia (Shaw et al. 2007), tradicionalmente se ha descrito como un depredador generalista y oportunista (Emmons 1987, Beck et al. 2005) aunque recientemente se ha evidenciado que en localidades específicas tiende a ser especialista (Monroy-Vilchis et al. 2009), consume incluso carroña en sitios donde las presas son escasas o donde la competencia es alta (Murphy et al. 1998, Bauer et al. 2005). El puma como carnívoro, representa uno de los grupos clave en el funcionamiento de ecosistemas naturales (Terborgh 1988) ya que mantiene el equilibrio entre las poblaciones naturales de presas y su ecosistema (Payán 2004); es además un posible indicador de presencia y abundancia de sus presas y del buen estado de conservación del ecosistema (Hornocker 1970, Noss et al. 1996). Este tipo de control sobre la densidad de las poblaciones de sus presas (top down), tiene un impacto a su vez en los patrones de dispersión, composición y estructura de los bosques lluviosos (Laundré \& Hernández 2010) lo que proporciona además, una justificación científica importante para conservar a esta especie (Noss et al. 1996, Berger 1997).

Los hábitos alimentarios del puma varían también con la latitud (Iriarte et al. 1990), de tal manera que en hábitats templados consumen presas grandes y tienden a ser especialistas, mientras que en hábitats tropicales, consume un mayor número de presas principalmente pequeñas (Monroy-Vilchis et al. 2009). Los estudios de hábitos alimentarios del puma que han sido conducidos principalmente en el norte del continente Americano (Toweill \& Meslow 1977, Anderson 1983, Currier 1983, Ackerman et al. 1984, Sweitzer et al. 1997, Pierce et al. 2000, Logan \& Sweanor 2001, Anderson \& Lindzey 2003, Laundré \& Hernández 2003, Leberg et al. 2004, Beck et al. 2005) confirman que casi todas sus presas son artyodactilos que pesan más de los $6.1 \mathrm{~kg}$; en cambio, el peso promedio de presas del puma en las regiones neotropicales es menor de los $6.1 \mathrm{~kg}$ (MonroyVilchis et al. 2009).

En Centro y Suramérica existen algunas investigaciones sobre la dieta del puma; México ha registrado importantes estudios, entre ellos en México central (De la Torre \& De la Riva 2009, Monroy-Vilchis et al. 2009), en Jalisco (Nuñez et al. 2000) y en Sonora (Rosas-Rosas et al. 2003); otros en Guatemala (Novack et al. 2005), en Argentina (Branch et al. 1996, Pessino et al. 2001), Brasil (Cascelli 2008), Chile (Yáñez et al. 1986, Courtin et al. 1980, Iriarte 1990, Rau et al. 1991, Franklin et al.1999, Rau \& Jiménez 2002) y Paraguay (Taber et al. 1997) entre otros estudios en donde se han hecho importantes avances, sin embargo, no hay ningún estudio de poblaciones de puma de los páramos y bosques altoandinos.

A pesar de que la dieta del puma es conocida en otros países, en Colombia solo existe información de algunas presas halladas en excretas de esta especie (Payán 2004) pero no se ha realizado ningún estudio sistemático sobre dieta. Por lo tanto, es muy importante adelantar estudios sobre los hábitos alimentarios de la especie en diferentes ecosistemas colombianos. En el presente trabajo se describe, por primera vez para Colombia, los hábitos alimentarios de los pumas estudiados en el Parque Nacional Natural Puracé y su variación durante tres años de estudio 2007-2009, particularmente en ecosistemas de páramo y bosque altoandino en donde no se ha realizado ninguna investigación en pumas.

\section{MATERIALES Y MÉTODOS}

El Parque Nacional Natural Puracé se encuentra ubicado en los Andes colombianos, entre los departamentos de Cauca y Huila, con alturas entre $1750-4700 \mathrm{msnm}$ y con una extensión de 83 000ha (UASPNN 2006). Fue declarado como Reserva de la Biosfera del cinturón Andino, y se convirtió en un área que protege ecosistemas vitales de la Nación y del Mundo (Myers 1988). El lugar en donde se recolectaron las muestras corresponde a la 
vertiente oriental de la cordillera central, en la zona sur del parque (01 56 ' $38^{\prime \prime}-01^{\circ} 55^{\prime} 11^{\prime \prime}$ $\mathrm{N}$ - 76035'39"-76³5'26" W). Allí se encuentra localizado el nacimiento de dos de los ríos más importantes del país, el Magdalena y el Caquetá.

En esta área el régimen de lluvias es de tipo bimodal. La época de mayor pluviosidad es entre abril y agosto, predominando los períodos secos de diciembre a febrero. La precipitación oscila entre $1200-2500 \mathrm{~mm}$. La temperatura promedio es de $10^{\circ} \mathrm{C}$, con nubosidad alta y brillo solar bajo. La evaporación está por debajo de 66mm (UAESPNN 2006).

El área de estudio se caracteriza por tener ecosistemas de bosque andino, bosque alto andino y de páramo principalmente, en donde se promueve una alta especiación y un alto endemismo debido a la adaptación de las especies de fauna y flora a las condiciones bióticas y abióticas específicas del área (Luteyn \& Baslev 1992, Buytaert et al. 2006). Los páramos se caracterizan por estar asociados a frailejones (Espeletia sp.) y pajonales (Calamagrostis sp., Puya sp.), mientras que el bosque andino y bosque alto andino, se asocia con especies como romerillo (Gynoxis sp.), chilco (Senecio andicola), mortiño (Hesperomeles meleslanuginosa), roble (Quercus humboldtii), aguacatillo (Ocotea catophytla), encenillo (Weinmania sp.) y pino colombiano (Podocarpus oleifelius) (Van der Hammen 1998). Estos ecosistemas altoandinos además de ser de gran importancia ecológica en la captación de carbono y en la producción y regulación de agua (Luteyn \& Baslev 1992), son ecosistemas extremadamente vulnerables a la intervención antrópica y representan los hábitats indispensables para las especies amenazadas de mamíferos emblemáticos como: puma (P. concolor), oso de anteojos (Tremarctos ornatus), danta de páramo (Tapirus pinchaque) y venado conejo (Pudu mephistophiles) entre otros.

Se recolectaron excrementos en tres periodos (de julio a noviembre 2007; de abril a mayo 2008 y de febrero a junio 2009) (Fig. 1). Las heces fueron identificadas según Aranda (2000), además se registró su posición geográfica, fecha y altitud. Posteriormente, los excrementos fueron lavados sobre un tamiz con el

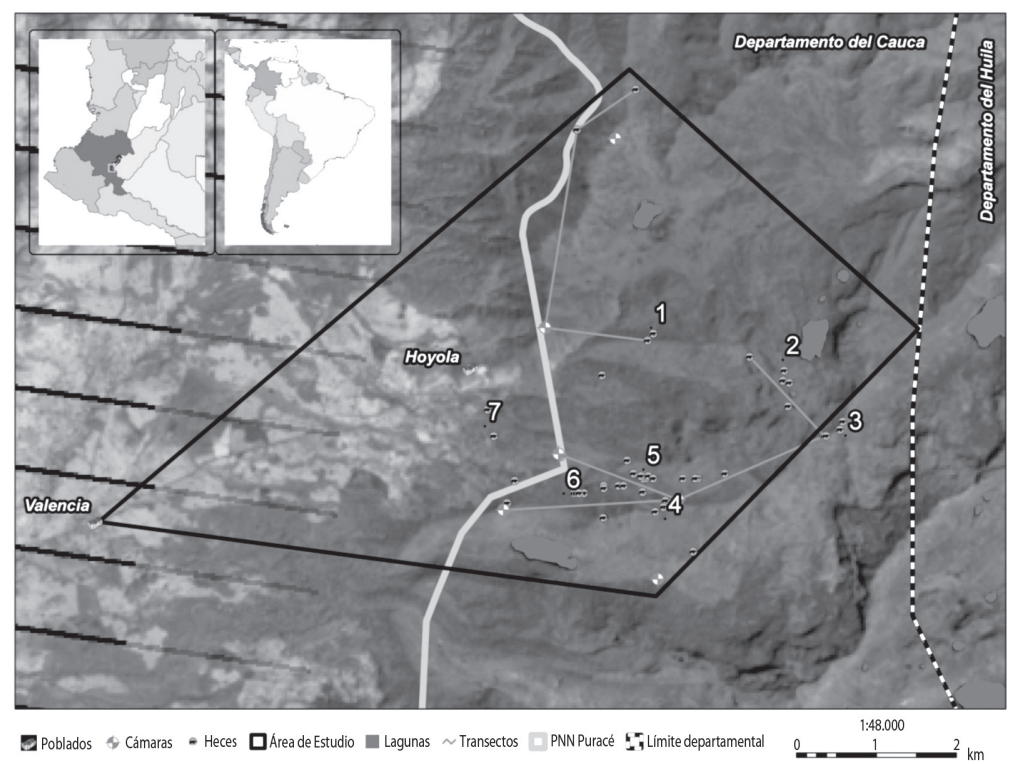

Fig. 1. Cámaras-trampa y excretas halladas. Los números indican las letrinas usadas por pumas.

Fig. 1. Camera traps location and puma scats founded. The numbers indicate the latrines used by pumas. 
fin de separar sus componentes (pelos, huesos, garras y dientes) (Ackerman et al. 1984). La identificación de los contenidos se realizó de dos formas: los huesos, garras y dientes fueron comparados con los ejemplares de la colección del Museo de Historia Natural de la Universidad del Cauca (MHNUC) y por la identificación de los patrones cuticulares y medulares de las muestras de pelos (Vásquez et al. 2000, Baca-Ibarra \& Sánchez-Cordero 2004) comparándolas con las muestras obtenidas de los ejemplares de la colección de referencia del MHNUC previamente preparadas.

Se estimó el número mínimo de excretas necesario para describir la dieta del puma, en el que se selecciona al azar una muestra de 10 excrementos y se compara la frecuencia de especies presa en la muestra con la frecuencia de especies presa del total de excrementos usando la prueba $\mathrm{X}^{2}$. En el caso de no encontrar diferencias estadísticas significativas, se selecciona otra muestra al azar de cinco excretas y se compara la respectiva frecuencia de las especies presa en las 15 excretas, con el total de excretas recolectadas $(n=60)$. Este proceso se repite hasta que la frecuencia en la muestra no difiera significativamente de la frecuencia en el total de muestras. Posteriormente, se llevó a cabo una estimación análoga del número de excrementos necesarios para describir la dieta del puma en el área de estudio, mediante una curva de acumulación de especies presa (Monroy-Vilchis et al. 2009).

Se calculó la frecuencia de presencia y la biomasa relativa consumida para cada especie presa (Ackerman et al. 1984) y adicionalmente se calculó el número mínimo de presas consumidas (Monroy-Vilchis et al. 2009), además se calculó la amplitud del nicho trófico, mediante la ecuación de Levins (1968): $\mathrm{B}=1 / \sum \mathrm{Pi}^{2}$, donde $P i$ es la proporción relativa de las presas de la categoría $i$ en la dieta. Posteriormente, para realizar comparaciones con otros estudios, se estandarizó la amplitud del nicho trófico de la siguiente forma: $\mathrm{B}_{\text {sta }}=\mathrm{B}-1 / \mathrm{n}-1$, donde $\mathrm{B}$ es el valor de la amplitud del nicho trófico observado y $\mathrm{n}$, es el total de presas usadas como recurso en la dieta (Krebs 1999).
Como una herramienta más para identificar la identidad de los excrementos y las posibles presas presentes en el área, se instalaron seis cámaras-trampa (Deer-cam 300) entre abril y junio de 2009 sobre los senderos identificados como usados por el puma.

\section{RESULTADOS}

Se identificaron cinco senderos usados permanentemente por puma durante el muestreo y se reconocieron siete letrinas principales sobre los cuales se halló el mayor número de heces. Durante las visitas, no siempre se encontraron heces en las letrinas pero si fueron usadas por el puma durante todo el muestreo al igual que los senderos (Cuadro 1).

Se recolectó un total de 60 excretas de puma, cubriendo $170.72 \mathrm{~km}$ efectuando recorridos exploratorios y preestablecidos en lugares con presencia histórica y rastros evidentes de la especie.

Cinco especies fueron identificadas en la dieta del puma; tres especies de mamíferos: venado soche (Mazama rufina), coatí de páramo (Nasuella olivacea), pudú ( $P$. mephistophiles) y el ave tinamú (Nothocercus julius), fueron reconocidas por fragmentos de hueso, cascos, dientes y/o garras, mientras que el conejo (Sylvilagus brasiliensis), fue reconocido mediante el análisis de patrones medulares y cuticulares en una sub-muestra de 1600 pelos extraída de 32 muestras del total de 60 excretas. Se encontraron pelos de puma en 30 de las 60 excretas, probablemente por su actividad de limpieza. Al microscópio óptico y con un aumento entre $40 \mathrm{X}$ y $100 \mathrm{X}$, no fue posible identificar diferencias entre pudú y venado soche, por lo que su presencia se asumió como venado desconocido.

En las 60 excretas de puma, se identificó un total de 54 ítems alimenticios macroscópicos, de los cuales el $81.7 \%$ corresponde a mamíferos, $11.7 \%$ vegetación y $1.7 \%$ aves. El número mínimo de excretas requeridas para determinar la dieta de pumas en esta área fue 10 usando el análisis de frecuencias $\left(\mathrm{X}^{2}=36\right.$, 
CUADRO 1

Letrinas usadas por $P$. concolor, por fecha de muestreo

TABLE 1

Letrines used by $P$. concolor, per sampling date

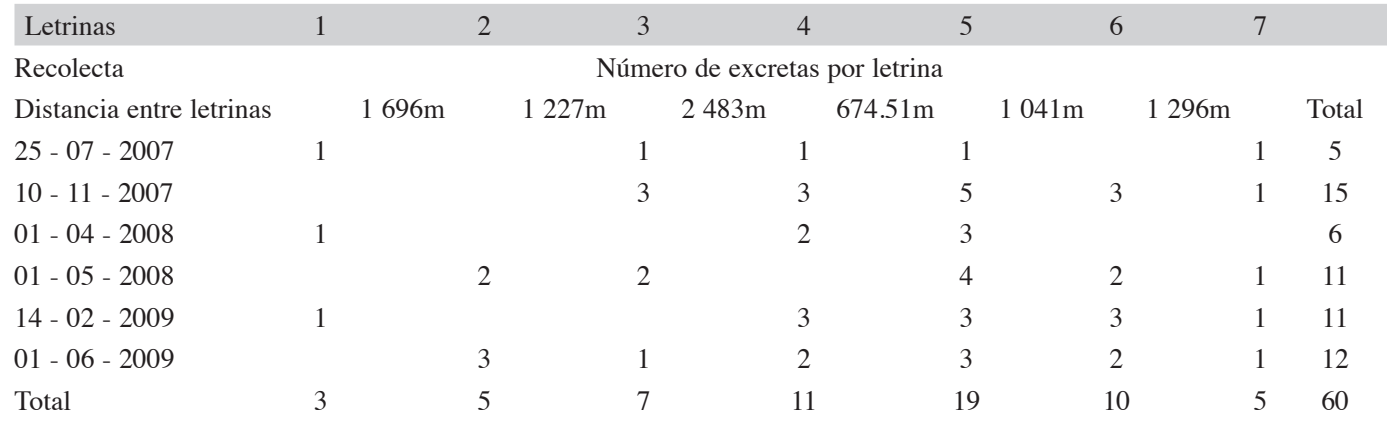

$\mathrm{p}>0.05, \mathrm{~g} .1 .=9)$ y 20 usando la curva de acumulación de especies.

La frecuencia de ocurrencia del venado conejo fue la más alta, seguida del coatí de páramo (Cuadro 2). En cuanto al porcentaje de biomasa relativa consumida, este estudio resalta la importancia de los venados, principalmente el pudú $(59.7 \%)$, como fuente principal de alimento del puma de páramo, seguido por el coatí de páramo (31.8\%) y el venado no identificado (29.5\%) (Cuadro 2).

$\mathrm{Al}$ calcular el número relativo de organismos consumidos de acuerdo con Ackerman et al. (1984), se obtiene que la presas mayormente consumidas por el puma son los coatíes (57.4), seguido del pudú (32.3), en menor número se encuentra el venado no identificado (9.9), conejo (6.6), tinamú (2.2) y venado soche (1.4) (Cuadro 2).

Con respecto al nicho trófico estandarizado se obtuvo un valor de 0.45 , lo cual muestra una tendencia especialista en el área de estudio.

Con un esfuerzo total de 354 trampasnoche se identificó la presencia de un macho adulto y una hembra adulta de puma en la zona entre otros mamíferos como: oso de anteojos (Tremarctos ornatus), danta de páramo (Tapirus pinchaque), pudú y un ave: gallinago (Gallinago nobilis).

\section{DISCUSIÓN}

Este es uno de los primeros trabajos sobre hábitos alimentarios de puma en las montañas de los Andes de Sur América y el único publicado para Colombia hasta el momento. El presente estudio evidencia una relación de dependencia íntima con el pudú por parte de los pumas estudiados, un venado amenazado y poco conocido presente en los ecosistemas de alta montaña de los Andes (Hershkovitz 1982, Wemmer 1998). Esta especie de venado tiene la mayor restricción en área de distribución, sus poblaciones están en disminución debido a la cacería humana, depredación por perros domésticos y pérdida de hábitat, lo cual lo categoriza como una especie casi amenazada (Hershkovitz 1982, Wemmer 1998, RodríguezMahecha et al. 2006). Este es el tercer estudio donde se evidencia la relación entre el puma y el pudú, ya que Courtin et al. (1980) y Rau et al. (1991) lo encontraron en excretas de pumas del sur de Chile. Por lo tanto, el pudú parece ser una presa base en cuanto a la distribución latitudinal Andina del puma y éste predador podría estar actuando como regulador de las poblaciones de pudú. Esto es entonces, una relación hábitat-específico para los Andes y fundamental para la conservación de ambas 
CUADRO 2

Componentes de la dieta de P. concolor en el Parque Nacional Natural Puracé

TABLE 2

P. concolor diet components in the Purace National Park

\begin{tabular}{|c|c|c|c|c|c|c|c|c|c|c|}
\hline PRESAS & $\begin{array}{l}\text { FA } \\
\text { (a) }\end{array}$ & $\begin{array}{l}\text { FO } \\
\text { (b) }\end{array}$ & $\begin{array}{c}\text { Peso } \\
\text { presa } \\
(\mathrm{g} .)\end{array}$ & $\begin{array}{l}\text { Peso } \\
(\mathrm{Kg}) \\
(\mathrm{c})\end{array}$ & $\begin{array}{l}\text { Factor } \\
\text { corrección } \\
\text { (d) }\end{array}$ & $\begin{array}{l}\text { BRC } \\
\text { (e) }\end{array}$ & $\begin{array}{c}\text { FO } \\
\text { (Factor de } \\
\text { corrección) } \\
\left(b_{\times} d\right)\end{array}$ & $\begin{array}{l}\% \text { de } \\
\text { BRC } \\
\text { (f) }\end{array}$ & $\begin{array}{l}\text { BRC / } \\
\text { peso } \\
(\mathrm{f} / \mathrm{c})\end{array}$ & $\begin{array}{l}\text { NROC } \\
(g)\end{array}$ \\
\hline \multicolumn{11}{|l|}{ MAMMALIA } \\
\hline \multicolumn{11}{|l|}{ Artiodactyla } \\
\hline Pudu mephistophiles & 34 & 56.7 & 5000 & 5 & 2.155 & 0.5975 & 122.1167 & 59.758 & 11.95 & 32.3495 \\
\hline Mazama rufina & 3 & 5 & 11000 & 11 & 2.365 & 0.0578 & 11.825 & 5.7866 & 0.53 & 1.42387 \\
\hline Venado no dentificado & 16 & 26.7 & 8000 & 8 & 2.26 & 0.2949 & 60.267 & 29.491 & 3.68 & 9.97814 \\
\hline \multicolumn{11}{|l|}{ Carnívora } \\
\hline $\begin{array}{l}\text { Nasuella olivacea } \\
\text { Lagomorpha }\end{array}$ & 26 & 43.3 & 1500 & 1.5 & $1.5^{*}$ & 0.3180 & 65 & 31.808 & 21.21 & 57.3964 \\
\hline $\begin{array}{l}\text { Silvilagus brasiliensis } \\
\text { AVES }\end{array}$ & 3 & 5 & 965 & 0.965 & $0.965^{*}$ & 0.0236 & 4.825 & 2.3611 & 2.45 & 6.62266 \\
\hline \multicolumn{11}{|l|}{ Tinamiforme } \\
\hline $\begin{array}{l}\text { Nothocercus julius } \\
\sum\end{array}$ & 1 & 1.7 & 350 & 0.35 & $0.35 *$ & 0.0028 & $\begin{array}{c}0.583 \\
204.35\end{array}$ & 0.2854 & $\begin{array}{c}0.81 \\
36.94\end{array}$ & 2.20755 \\
\hline
\end{tabular}

(a) Frecuencia Absoluta=número total de heces que contienen una especie determinada; (b) Frecuencia de Presencia=porcentaje de heces que contienen los ítems de las presas; (c) Peso promedio de las presas tomado de Tirira (2007) y Rodríguez-Mahecha et al. (2006); (d) Factor de corrección de Ackerman et al. (1984), Y=1.98+0.035X; (e) Biomasa Relativa Consumida $=\left\{(\mathrm{b} . \mathrm{d}) / \sum\right.$ (b.d $\left.)\right\}$; (g) Número Relativo de Organismos Consumidos $=\{(\mathrm{f} / \mathrm{c}) / \Sigma(\mathrm{f} / \mathrm{c})\}$; *, el factor de corrección no se aplica.

especies, dada su interdependencia. Al procurar la conservación in situ del puma, se conserva de manera indirecta a sus presas más importantes (Terborgh 1988) y a su vez, a las plantas y animales que los alimentan siendo de esta manera otra justificación importante para conservar especies amenazadas como el pudú. El presente estudio evidencia especies nuevas en la dieta del puma, lo que constituye una evidencia más de la flexibilidad de su comportamiento y de la variación de los hábitos alimentarios existente en los diferentes ecosistemas a lo largo de su distribución.

La dieta de los pumas estudiados en el PNN Puracé, está compuesta principalmente por mamíferos medianos, siendo los venados (Artiodactyla) y los coatíes (Carnívora), las presas más importantes y de mayor aporte con base en la biomasa relativa consumida y la frecuencia de ocurrencia. En este sentido, este estudio concuerda con otras investigaciones sobre los hábitos alimentarios del puma, en los que se reconoce la importancia de los ungulados como sustento principal y la de otras especies de medianos mamíferos y aves dentro de su dieta, haciendo del puma una especie generalista y oportunista considerando toda su distribución (Hornocker 1970, Rau et al. 1991, Beck et al. 2005, Monroy-Vilchis et al. 2009). Por otra parte, los registros de presas halladas en excrementos de puma en el departamento del Quindío-Colombia, por Payán (2004), señalan a Choloepus hoffmani, Tamandua mexicana y Mazama sp., como especies importantes en la dieta de los pumas en la cordillera central, coincidiendo en el género Mazama, pero con ausencia de pudú. Esto se podría explicar por el menor tamaño de la muestra analizada 
por Payán (2004). Es interesante resaltar, el contraste con el resto de especies, dado que la composición y disponibilidad de presas, varía según la distribución geográfica (latitud, altitud) y tipos de ecosistemas (Iriarte 1990).

Adicionalmente, los venados y los coatíes presentes en el área son presas que pueden satisfacer las necesidades energéticas diarias de los pumas (Monroy-Vilchis et al. 2009). Teniendo en cuenta el tamaño corporal de estas especies y que el peso es aproximado a $5 \mathrm{~kg}$, es posible asumir que el esfuerzo y el costo energético sea quizás mayor en estas zonas de páramo que en sectores colombianos por debajo de los 3 $000 \mathrm{msnm}$ con presencia de presas más grandes y con mayor peso (e.g. Odocoileus virginianus) así como se menciona en otros estudios donde la disponibilidad de alimento es mayor (Hornocker 1970, Ackerman et al. 1984, Yañez et al. 1986, Pessino et al. 2001, Branch et al. 1996, Taber et al. 1997, Franklin et al. 1999, Nuñez et al. 2000, Rau \& Jiménez 2002, Novack et al. 2005, Monroy-Vilchis et al. 2009).

Con base en el cálculo del número relativo de organismos consumidos (Ackerman et al. 1984), el coatí de páramo y el pudú son las dos especies más importantes, resultando como ocasionales otras presas pequeñas y medianas como venado soche, conejo y tinamú.

Por otra parte, Nuñez et al. (2000), basados en una muestra total de 65 heces de puma, estimaron un mínimo de 35 excrementos con el uso el test de $\mathrm{X}^{2}$ y de 50 excrementos mediante la curva de acumulación de especies, suficientes para determinar la dieta de la especie hasta tal punto en que heces adicionales no tendrían efecto sobre los resultados; sin embargo, como puede pasar en los trópicos húmedos o en regiones con mayor número y disponibilidad de presas, una mayor cantidad de heces puede ser requerida. Recientemente, Monroy-Vilchis et al. (2009), estimaron que un mínimo de 15 excrementos es suficiente para describir la dieta de pumas en México Central $(n=104)$. Para el presente estudio, la prueba de $\mathrm{X}^{2}$ mostró que un mínimo de 10 excrementos es suficiente para describir la dieta del puma y de 20 excrementos mediante el uso de la curva de acumulación de especies no se presentan cambios en la composición de la dieta, lo que nos permite inferir que en las 60 excretas recolectadas en tres periodos diferentes (2007, 2008 y 2009), se encuentran representadas las presas más importantes para los pumas estudiados en los ecosistemas de páramo y bosque altoandino presentes en área de estudio. No obstante, con el seguimiento de este estudio a través de los años incluyendo nuevos sectores a diferentes altitudes, se podría entender mejor las tendencias, la variación y la importancia de ciertas presas, así como reconsiderar el número mínimo de muestras que puede ser requerido para monitorear y determinar los hábitos alimenticios del puma anualmente. La discrepancia entre los resultados obtenidos por Nuñez et al. (2000), Monroy-Vilchis et al. (2009) y este estudio, puede explicarse por la diversidad de presas disponibles en las diferentes áreas en donde se han conducido las investigaciones.

Este comportamiento en la dieta de los pumas presentes en el área de estudio coincide con algunos trabajos desarrollados en el sur de Chile (Iriarte et al. 1990, Rau et al. 1991, Rau \& Jimenez 2001) y en Argentina (Branch et al. 1996, Pessino et al. 2001), en donde se encontró que la selección y depredación estaba más enfocada en una especie; sin embargo contrasta con los resultados de las demás investigaciones sobre la dieta del puma.

El valor de nicho trófico estandarizado obtenido (0.45) nos indica que el puma en la zona tiene tendencia especialista, esto era un tanto esperable ya que en la zona de estudio el puma se alimenta de muy pocas presas (6), siendo uno de los lugares con menor número de presas. Debido a las características de altitud y al relativo buen estado de conservación de la zona de estudio, los pumas estudiados podrían estar presentando una especialización como lo sugerido por Monroy-Viclhis et al. (2009). Otra interpretación es que los pumas estudiados muestran una adaptación a tomar los recursos de presas en la medida de su disponibilidad como lo evidenció Emmons (1987). Estas inferencias pueden ser validadas en un futuro estudio donde se evalúe la disponibilidad y 
abundancia de presas, ya que es difícil hablar de selección por parte de los pumas estudiados sin tener conocimiento de la abundancia de presas en el área. La pequeña muestra espacial del presente estudio limita la información a unos cuantos individuos de puma y hace difícil las generalizaciones e inferencias.

La diversidad de los ecosistemas juega un papel determinante en la variación y selección de las presas de puma a lo largo de su distribución, como se evidenció aquí para los hábitats de páramo y bosque altoandino. Si se tiene en cuenta que no existen registros sobre ataques de pumas a dantas de montaña en esta región, y la ausencia de grandes mamíferos en la dieta de los pumas aquí estudiados, se podría explicar la mayor tendencia selectiva y depredadora sobre el pudú y el coatí, lo que convierte a estas dos especies en los recursos disponibles más importantes para el puma de páramo. Esto sugiere que el bajo número de especies presa de las poblaciones de puma de páramo implica poblaciones menos resistentes a cambios en la composición de especies que otros pumas con gremios de especies presas más abundantes. El hecho de que las especies presa más importantes sean especies vulnerables y desconocidas, hace que las amenazas sobrepasen su efecto directo sobre cada una y afecten otras especies que dependen de ellas. Esto también resalta la necesidad de diseñar estrategias de manejo y conservación para ecosistemas únicos, importantes y frágiles como el páramo (Luteyn \& Baslev 1992, Buytaert et al. 2006) y el uso de especies focales en gremio. Por lo tanto, los Parques Nacionales Naturales en páramos Andinos en Colombia representan un recurso de conservación importante para las poblaciones de pumas andinos y sus presas tan particulares como amenazadas.

\section{AGRADECIMIENTOS}

Estamos muy agradecidos con Panthera Colombia; María Teresa Amaya, administradora del Parque Puracé y especialmente con el guardaparque Gustavo Adolfo Papamija, quien dió una gran contribución a esta investigación.
Apreciamos los aportes de Héctor Ramírez en la identificación del material óseo; Fernando Muñoz en la elaboración del mapa, Pilar Rivas, directora del Museo de Historia Natural, Universidad del Cauca; Andrés Torres director de la Unidad de Microscopia, Universidad del Cauca y especialmente a Patricia Mosquera por el apoyo brindado para la toma de fotografías de las muestras de pelos.

\section{RESUMEN}

La dieta de Puma concolor es ampliamente conocida a lo largo de su distribución, sin embargo, en Colombia no se ha realizado ningún estudio sobre sus hábitos alimentarios. Entre 2007-2009, la dieta de puma fue analizada en el Parque-Nacional-Natural-Puracé, sur occidente de los Andes colombianos. Ítems alimenticios de cinco especies presa fueron identificadas en su dieta; el venado conejo ( $P u d u$ mephistophiles) es la presa más importante. Como herramienta complementaria para la identificación de huesos y pelos contenidos en heces $(n=60)$, se instalaron seis cámarastrampa en lugares estratégicos, para registrar la presencia de pumas y presas potenciales. El descubrimiento de la dependencia de los pumas con el pudú sugiere una única adaptación de los pumas de paramo a la disponibilidad de presas y resalta su importancia como reguladores de las poblaciones presa. Estos resultados contribuyen a incrementar el poco conocimiento sobre la ecología de pumas de los Andes, de sus presas y de las especies en su conjunto en Colombia. Obtener información sobre el grupo de presas de pumas en diferentes ecosistemas, es esencial para entender los requerimientos regionales para su supervivencia y diseñar acciones de conservación que permitan seguir/evaluar las necesidades particulares de áreas protegidas en toda su distribución.

Palabras clave: Andes colombianos, cámaras trampa, dieta, Parque Nacional Puracé, presas, Pudú, Puma concolor.

\section{REFERENCIAS}

Ackerman, B.B., F.G. Lindzey \& T.P. Hemker. 1984. Cougar food habits in southern Utah. J. Wildlife Manage. 48: 147-155.

Anderson, A.E. 1983. A critical review of literature on puma (Felis concolor). Colorado Div. Wildl. Spec. Rep. 54.

Anderson, C.R. \& F.G. Lindzey. 2003. Estimating Cougar Predation Rates from GPS Location Clusters. J. Wildlife Manage. 67: 307-316. 
Aranda, M. 2000. Huellas y otros rastros de los mamíferos grandes y medianos de México. Instituto de Ecología, A.C. Xalapa, Veracruz, México.

Baca-Ibarra, I.I. \& V. Sánchez-Cordero. 2004. Catálogo de pelos de guardia dorsal en mamíferos del estado de Oaxaca, México. Anales del Instituto de Biología, UNAM, Serie Zool. 75: 383-437.

Bauer, J.W., K.A. Logan, L.L. Sweanor \& W.M. Boyce. 2005. Scavenging Behavior in Puma. Southwest Nat. 50: 466-471.

Beck, T., J. Beecham, P. Beier, T. Hofstra, M. Hornocker, F. Lindzey, K. Logan, B. Pierce, H. Quigley, H. Shaw, R. Sparrowe \& S. Torres. 2005. Cougar Management Guidelines Working Group. WildFutures, Bainbridge Island, Washington, EEUU.

Berger, J. 1997. Population constraints associated with the use of black rhinos as an umbrella species for desert herbivores. Conserv. Biol. 11: 69-78.

Branch, L.C., M. Pessino \& D. Villarreal. 1996. Response of pumas to a population decline of the plains Vizcacha. J. Mammal. 77: 1132-1140.

Buytaert, W., R. Célleri, B. De Biévre, F. Cisneros, G. Wyseure, J. Deckers \& R. Hofstede. 2006. Human impact on the hydrology of the Andean paramos. Earth Sci. 79: 53-72.

Cascelli de Acevedo, F. 2008. Food Habits and Livestock Depredation of Sympatric Jaguars and Pumas in the Iguaçu National Park, South Brazil. Biotropica 40: 494-500.

Caso, A., C. Lopez-Gonzalez, E. Payan, E. Eizirik, T. de Oliveira, R. Leite-Pitman, M. Kelly, C. Valderrama \& M. Lucherini. 2008. Puma concolor. IUCN Red List of Threatened Species, IUCN, Gland, Switzerland. (Consultado 20 de enero 2011, www.iucnredlist.org).

Courtin, S.L., N.V. Pacheco \& W.D. Eldridge. 1980. Observaciones de alimentación, movimiento y preferencia de hábitat del puma, en el Islote Rupanco, Chile. Medio Ambiente 4: 50-55.

Culver, M., W.E. Jhonson, J. Pecon-Slattery \& J. O'Brien. 2000. Genomic ancestry of the American puma (Puma concolor). J. Hered. 9: 186-197.

Currier, M.J.P. 1983. Felis concolor. Mamm. Species 1: $1-7$.

De la Torre, A.J. \& G. De la Riva. 2009. Food habits of pumas (Puma concolor) in a semiarid region of central Mexico. Mastozool. Neotrop. 16: 211-216.
Emmons, L.H. 1987. Comparative feeding ecology of felids in a neotropical rainforest. Behav. Ecol. Sociobiol. 20: 271-283.

Franklin, W.L., W.E. Johnson, R.J. Sarno \& J.A. Iriarte. 1999. Ecology of the Patagonia puma Felis concolor patagonica in southern Chile. Biol. Conserv. 90: 33-40.

Hershkovitz, P. 1982. Neotropical deer (Cervidae), Part I, Pudus, Genus Pudu Gray. Fieldiana: Zool. 11:1-86.

Hornocker, M.G. 1970. An Analysis of Mountain Lion Predation upon Mule Deer and Elk in the Idaho Primitive Area. Wildlife Monogr. 21: 3-39.

Iriarte, J.A., W.L. Franklin, W.E. Johnson \& K.H. Redford. 1990. Biogeographic variation of food habits and body size of the America puma. Oecologia 85: $185-190$

Kelly, M., A. Noss, M. Di Bitetti, L. Maffei, R. Arispe, A. Paviolo, C. De Angelo \& Y. Di Blanco. 2008. Estimating Puma densities from camera trapping across three study sites: Bolivia, Argentina and Belize. J. Mammal. 89: 408-418.

Krebs, C.J. 1999. Ecological methodology. Cummings, Nueva York, EEUU.

Laundré, J.W. \& L. Hernández 2003. Winter hunting habitat of Pumas (Puma concolor) in northwestern Utah and southern Idaho, USA. Wildlife Biol. 9: 123-129.

Laundré, J.W. \& L. Hernández 2010. What we know about pumas in Latin America, p. 76-90. In M.G. Hornocker \& S. Negri (eds.). 2010. Cougar: ecology and conservation. University of Chicago, Chicago, EEUU.

Leberg, P., M. Carloss, L. Dugas, K. Pilgrim, L. Scott, C. Green \& D. Scognamillo. 2004. Recent record of a Cougar (Puma concolor) in Louisiana, with notes on diet, based on analysis of fecal materials. Southeast Nat. 3: 653-658.

Logan, K. \& L. Sweanor. 2001. Desert puma, evolutionary, ecology and conservation of an enduring carnivore. Island, Washington D.C., EEUU.

Luteyn, J.L \& H. Baslev. 1992. Paramos: Why study them? p. 1-14. In H. Baslev \& J.L. Luteyn (eds.). Paramo: An Andean ecosystem under human influence. Academic, Londres, Inglaterra.

Monroy-Vilchis, O., Y. Gómez, M. Janczur \& V. Urios. 2009. Food Niche of Puma concolor in Central Mexico. Wildlife Biol. 15: 97-105. 
Murphy, K.M., G.S. Felzien, M.G. Hornocker \& T.K. Ruth. 1998. Encounter Competition between Bears and Cougars: Some Ecological Implications. A Selection of Papers from the Tenth International Conference on Bear Research and Management, Fairbanks, Alaska, July 1995, and Mora, Suecia. Ursus 10: 55-60.

Myers, N. 1988. Theratened biotas: hotspots in tropical forest. Environmentalist 8: 1-20.

Noss, R.F., H.B. Quigley, M.G. Hornocker, T. Merril \& P.C. Paquet. 1996. Conservation Biology and Carnivore Conservation in the Rocky Mountains. Conserv. Biol. 10: 949-963.

Novack, A.J., M.B. Main, M.E. Sunquist \& R.F. Labisky. 2005. Foraging ecology of jaguar (Panthera onca) and puma (Puma concolor) in hunted and non-hunted sites within the Maya Biosphere Reserve, Guatemala. J. Zool. 267: 167-178.

Nowell, K. \& P. Jackson. 1996. Wild cats. Status survey and conservation action plan. IUCN/SSC Cat Specialist Group. Gland, Suiza.

Nuñez, R., B. Miller \& F. Lindzey. 2000. Food habits of jaguars and pumas in Jalisco, México. J. Zool. 252: 373-379.

Payán, E. 2004. Diagnóstico, análisis y propuestas de manejo para el conflicto de predación entre carnívoros y los sistemas productivos de la región Andina con énfasis en el eje cafetero de Colombia. Instituto Alexander von Humboldt, Bogotá, Colombia.

Pessino, M., J. Sarasola, C. Wander \& N. Besoky. 2001. Respuesta a largo plazo del puma (Puma concolor) a una declinación poblacional de la Viscacha (Lagostomus maximus) en el desierto del Monte, Argentina. Ecol. Austral 11: 61-67.

Pierce, B.M., B.C. Bleich \& R.T. Bowyer. 2000. Selection of mule deer by mountain lions and coyotes: effects of hunting style, body size and reproductive status. J. Mammal. 81: 462-472.

Rau, J.R., M. Tilleria, D. Martinez \& A. Muñoz. 1991 Dieta de Felis concolor (Carnívora: Felidae) en áreas silvestres protegidas del sur de Chile. Chil. Hist. Nat. 64: $139-144$

Rau, J.R. \& J.E. Jiménez. 2002. Diet of Puma (Puma concolor, Carnivora: Felidae) in Coastal and Andean Ranges of Southern Chile. Stud. Neotrop. Fauna Environ. 37: 201-205.

Rodríguez-Mahecha, J.V., M. Alberico, F. Trujillo \& J. Jorgenson. 2006. Libro rojo de los mamíferos de Colombia. Serie libros rojos de especies amenazadas de Colombia. Conservación Internacional, Bogotá, Colombia.
Rosas-Rosas, O.C., R. Valdez, L.C. Bender \& D. Daniel. 2003. Food habits of pumas in northwestern Sonora, México. Wildlife Soc. B. 31: 528-535.

Shaw, H.G., P. Beier, M. Culver \& M. Grigione. 2007. Puma field guide. A guide covering the biological considerations, general life history, identification, assessment and management of Puma concolor. The Cougar Network.

Soria-Díaz, L., O. Monroy-Vilchis, C. Rodríguez-Soto, M.M. Zarco-González \& V. Urios. 2010. Variation of abundance and density of Puma concolor in zones of high and low concentration of camera traps in Central Mexico. Anim. Biol. 60: 361-371.

Sweitzer, R.A., S.H. Jenkins \& J. Berger. 1997. Near extinction of porcupines by mountain lions and consequences of ecosystem change in the Great Basin desert. Conserv. Biol. 11: 1407-1417.

Taber, A.B., A.J. Novaro, N. Neris \& F.H. Colman. 1997. The food habits of Sympatric Jaguar and Puma in the Paraguayan Chaco. Biotropica 29: 204-213.

Terborgh, J. 1988. The big things that rule the world - a sequel to E.O. Wilson. Conserv. Biol. 2: 402-403.

Tirira, D. 2007. Guía de campo de los mamíferos del Ecuador. Ediciones Murciélago Blanco, Quito, Ecuador.

Toweill, D.E. \& E.C. Meslow. 1977. Food habits of Cougars in Oregon. J. Wildife Manag. 41: 576-578.

UAESPNN. 2006. Unidad administrativa especial de sistemas de Parques Nacionales Naturales. Plan de manejo del Parque Nacional Natural Puracé. Bogotá, Colombia.

Vásquez, D.E., P.G. Perovic \& A. De Olsen. 2000. Patrones cuticulares y medulares de pelos de mamíferos del Noroeste de Argentina (Carnívora y Dactirodactyla). Mastozool. Neotrop. 7: 141-147.

Van der Hammen, T. 1998. Páramos, p. 10-37. In M.E. Chaves \& N. Arango (eds.). Informe nacional sobre el estado de la biodiversidad 1997. Instituto de Investigación de recursos biológicos Alexander von Humboldt, Bogotá, Colombia.

Wemmer, C. 1998. Deer. Status Survey and Conservation Action Plan. IUCN/SSC Deer Specialist Group. IUCN, Gland, Suiza and Cambridge, Inglaterra.

Yáñez, J.L., J.C. Cárdenas, P. Gezelle \& F.M. Jaksić. 1986. Food Habits of the Southernmost Mountain Lions (Felis concolor) in South America: Natural versus Livestocked Ranges. J. Mammal. 67: 604-606. 